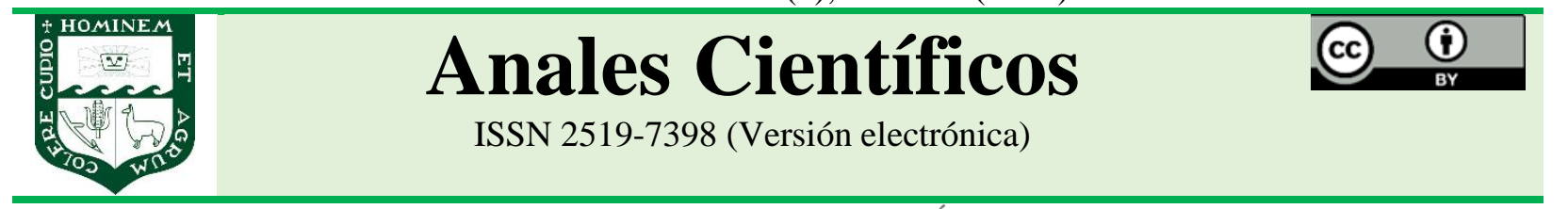

ARTÍCULO ORIGINAL - RESEARCH ARTICLE

http://dx.doi.org/10.21704/ac.v81i2.1640

\title{
VARIACIÓN EN LA DENSIDAD DE POBLACIONES DE Apis mellifera L. EN EL CULTIVO DE MANDARINA
}

\section{Variation in population density of Apis mellifera $L$. on tangerine crop}

\author{
Yessica Vargas ${ }^{1}$; Agustín Martos ${ }^{1 *}$; $;$ Julián Chura $^{1}$ \\ ${ }^{1}$ Facultad de Agronomía, Universidad Nacional Agraria La Molina, 15024, Lima, Perú. \\ * E-mail: amartos@lamolina.edu.pe
}

Recibido: 16/01/2020; Aceptado: 15/12/2020; Publicado: 31/12/2020

\begin{abstract}
The research was carried out in order to determine the variation in population density of Apis mellifera L. along blooming period of Satsuma Owari tangerine (Citrus unshiu Marc.), according to environmental factors at Huaral - Lima. Adults honey bee data were registered on 10 trees, three times a week, on schedules from 8:00 a.m. - 10:00 a.m., 1:00 p.m. - 3:00 p.m. and 3:00 p.m. - 5:00 p.m. on flower buds, opened flowers and withered flowers phases. Besides, aspects of adult bee behavior were registered. Low population values were registered on flower buds and withered flowers phases, while high values were registered on opened flowers phase by the presence of nectar. Higher densities of adult bees were registered at 11:00 a.m. - 1:00 p.m. and 3:00 p.m. - 5:00 p.m. schedules, because of good temperature and solar radiation conditions, while low values were registered on 8:00 a.m. - 10:00 a.m. schedule. The research indicates that tangerine compete against other fruit trees, respect to the visit of bees for harvest of pollen, nectar and pollination, on schedule from 11 a.m. forward on flowering period.
\end{abstract}

Keywords: Apis mellifera L.; honey bee; population density; insect behavior; tangerine.

\section{RESUMEN}

La investigación se realizó con el objetivo de determinar la variación en la densidad de poblaciones de Apis mellifera L. a lo largo del periodo de floración del mandarino Satsuma Owari (Citrus unshiu Marc.), de acuerdo con los factores medioambientales en Huaral, Lima, Perú. Datos de abejas adultas se tomaron sobre 10 árboles, tres veces por semana en horarios de 8:00 a.m. - 10:00 a.m., 11:00 a.m. - 1:00 p.m., y 3:00 p.m. - 5:00 p.m., en fases de botoneo, de flores abiertas y de flores con pétalos caídos y marchitos. También se registraron aspectos del comportamiento en abejas adultas. Los menores valores poblacionales fueron registrados en fases de botoneo y flores con pétalos caídos y marchitos, mientras que altos valores ocurrieron en fase de flores abiertas por la presencia de néctar. Las mayores densidades de abejas adultas fueron registradas en horarios de 11:00 a.m. - 1:00 p.m. y 3:00 p.m. - 5:00 p.m., debido a las buenas condiciones de temperatura y radiación solar, mientras que los menores valores fueron registrados de 8:00 a.m. - 10:00 a.m. La investigación indica que la mandarina compite con otros árboles frutales, respecto a la visita de abejas para recolección de polen, néctar y polinización, a partir de las 11 a.m. en temporada de floración.

Palabras clave: Apis mellifera L.; abeja melífera; densidad de poblaciones; comportamiento de insectos; mandarina. 


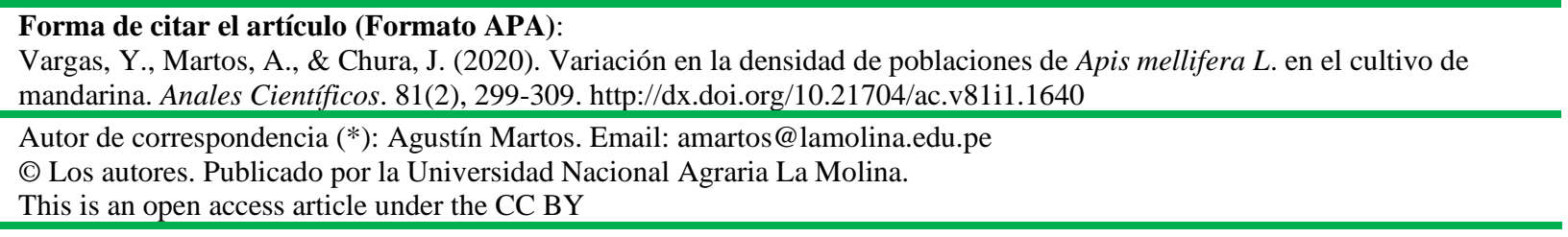

\section{INTRODUCCIÓN}

La abeja melífera (Apis mellifera L.) es uno de los insectos más importantes en la polinización de plantas cultivadas tales como el palto (Persea americana Mill.) y el arándano (Vaccinium corymbosum L.), entre otras, en el Perú. Según INDAP (2016) el polen se traslada de una flor a otra, en la mayoría de los casos, mediante insectos polinizadores que son atraídos por nectarios, olores, formas y colores; en este proceso, la abeja melífera aprovecha para recolectar néctar y polen, hecho que se magnifica ante una alta población de esta especie de insecto. Dadant (1979) menciona que el comportamiento de pecoreo de las abejas melíferas es adaptable a la cantidad de flores y su contenido en néctar y polen de las plantas, visitando flores en horarios variables según especies de plantas, afirmando que las flores de cítricos son nectaríferas. Osorio et al. (2002) afirman que los niveles de precipitación, temperatura, humedad relativa, brillo solar, al igual que la evapotranspiración, para las principales zonas biogeográficas, permiten considerar más fácilmente los periodos de floración y cosechas apícolas. En el mismo sentido, entre los factores que afectan la frecuencia de visitas de las abejas pecoreadoras están los aspectos climáticos y ambientales, la recompensa floral ofrecida por la planta visitada, la constancia floral hacia cierto tipo de recurso y los factores de atracción de las plantas; durante la mañana y el medio día hay una mayor frecuencia de visitas a la flora que en la tarde (Insuasty et al., 2015).

Las temperaturas y las lluvias afectan de modo importante el pecoreo de la abeja (Gómez y Esteban, 2016). En palto, la variedad Edranol tuvo mayor cantidad de abejas $/ \mathrm{m} 2 /$ minuto que la variedad Hass, debido a que Edranol tuvo mayor cantidad de flores que el cultivar Hass; sólo en Edranol existió una correlación positiva entre las flores abiertas y la cantidad de abejas (Castillo, 2002). Según Cautín (1996), citado por Castillo (2002), las abejas frecuentan los árboles de palto desde las 11:00 a las 14:00 horas, que es el tiempo en que el estado masculino y femenino de las flores tienen mayor posibilidad de traslape. Palacios (2005) refiere que la temperatura $\left(19\right.$ y $24^{\circ} \mathrm{C}$ para la apertura floral), la nutrición, disponibilidad de agua y la luz son importantes en el proceso de floración de los cítricos. Agrega que, en mandarinos que producen frutos con semillas tales como Kara, Murcott y Malvasio, la polinización cruzada no es problema, favoreciendo, por el contrario, la producción, siendo las abejas (53\%) los principales agentes polinizadores que resultan atraídas por el aroma intenso de las flores y la alta cantidad de néctar que producen.

Los cítricos, sin embargo, no requieren necesariamente de la participación de la abeja melífera u otro agente polinizador por su condición partenocárpica en muchos de los híbridos y cultivares comerciales como el mandarino 'Satsuma' (Citrus unshiu Marc.) (Amorós, 1999; Davies y Albrigo, 1999; Palacios, 2005). Machado (comunicación personal, 2015; fundo la Candelaria-Huaral-Lima) y Mendoza (comunicación personal, 2016; fundo la CandelariaHuaral-Lima), afirman que el mandarino Satsuma produce frutos sin semillas, aunque las flores sean visitadas y polinizadas por abejas. Avellaneda (2009) reporta que la naranja Valencia (Citrus sinensis) es visitada por las abejas melíferas en horario de 9:00 a.m. y 2:00 p.m.; en limón Tahití (Citrus aurantifolia) y el tangelo (Citrus reticulata x paradisi) son visitados a lo largo del día; sin embargo, C. aurantifolia es mayormente visitado a las 2:00 p.m. y el tangelo a las 5:00 p.m. VanEngelsdorp y Meixner (2010) y Bacandritsos et al. (2010) consideran a las plagas, cambio climático y pesticidas como factores que afectan a las poblaciones de abejas melíferas.

En América Latina, anualmente se pierden entre 13\% (Perú) y 53\% (Chile) de colmenas de abejas Apis mellifera por efecto de múltiples factores tales como el clima, aspecto sanitario y efectos de pesticidas (Repetto, 2018). En flores de melón la actividad colectora diaria de las abejas se inicia poco después de las seis de la mañana, con pocas visitas, y se va incrementando en los períodos siguientes; para el 
período de 9:30 a 10:30 a.m., se alcanza el clímax de visitas, donde se contabilizan algo más de un cuarto de las visitas diarias, casi todas para la recolección de polen; en el período de 11:00 a.m. a 12:00 m., se experimentó un descenso en la actividad, seguido de un repunte en el siguiente período, de 12:30 a 1:30 p.m.; a partir de ese período, la actividad declina, hasta ser casi nula a las 5 p.m.; la mayor parte de las visitas, casi la mitad (43\%), fueron realizadas entre 8:00 y10:30 a.m.; esto puede deberse a que las visitas por polen sólo se realizan hacia las primeras horas de la mañana, entre 7:00 y 11:00 a.m.; las visitas por néctar, en cambio, empezaron desde la mañana, en baja proporción y continuaron hasta entrada la tarde, cerca de las 5:00 p.m. (Di Trani, 2007). Las abejas prefieren colectar polen de mañana y néctar en la tarde; la mayor colecta de polen durante la mañana puede obedecer a necesidades de la colonia o a que el recurso es más fácil de extraer de las flores en las primeras horas del día (Santos y Invernizzi, 2018).

La presente investigación se realizó con el objetivo de determinar la variación en la densidad poblacional de Apis mellifera $L$. a lo largo del periodo de floración del mandarino Satsuma Owari (Citrus unshiu Marc.), de acuerdo a los factores medioambientales en Huaral Lima

\section{MATERIALES Y MÉTODOS}

Se realizaron evaluaciones de poblaciones de Apis mellifera en una plantación de 4 hectáreas de mandarino Satsuma Owari (Citrus unshiu Marc.) en floración en Huaral-Lima, de setiembre a noviembre del 2015, con una temperatura de 19,2oC (18,2 - 20,5), una humedad relativa de $82,9 \%(80-89)$ y $2,9(0-7,4)$ horas de sol, en promedio, respectivamente. La plantación de mandarina se dividió en diez áreas, eligiéndose un árbol en cada una de ellas. En total se seleccionaron 10 árboles sobre los cuales se hicieron 18 evaluaciones durante el periodo de floración.

Variación en la densidad de poblaciones de Apis mellifera $L$. por día y según horarios

En cada árbol de mandarino, sobre las flores, en cuatro puntos cardinales del mismo, se contó y registró el número de abejas melíferas en pecoreo por un tiempo de diez minutos. Las evaluaciones se realizaron tres veces por semana, en horarios de 8:00 a.m. - 10:00 a.m., 11:00 a.m. - 1:00 p.m., y 3:00 p.m. - 5:00 p.m. Las evaluaciones se hicieron en tres fases del desarrollo fenológico de la flor: botoneo, flores abiertas y flores con pétalos caídos y marchitos.

Comportamiento de Apis mellifera L. durante el pecoreo en flores de mandarino, mediante observaciones frecuentes a lo largo del día se registraron aspectos del comportamiento de la abeja melífera como: vuelo, visita a flores, pecoreo y recolección de néctar, horarios de visita, tiempo de visita por flor, recolección de agua y visita a flores de otras plantas cercanas al cultivo de mandarino.

\section{RESULTADOS Y DISCUSIÓN}

Variación en la densidad de poblaciones de Apis mellifera L. por día En la Tabla 1 se puede apreciar que el número promedio de abejas por árbol por día a lo largo del periodo de evaluación varió entre 1 a 110 abejas, según fecha de evaluación y fase de la floración en la plantación.

Los menores valores se registraron en las fases de botoneo (1 - 27 abejas por árbol) y de flores con pétalos caídos y marchitos ( 1 - 37 abejas por árbol), mientras que los mayores valores se dieron en la fase de flores abiertas (51 - 110 abejas por árbol). Dadant (1979), INDAP (2016) y Insuasty et al. (2015) refieren que los insectos y las abejas visitan las flores atraídas por el néctar que producen.

En la fase de botoneo, el número de abejas registradas fue bajo debido, al parecer, al escaso número de flores abiertas, nula oferta de néctar y probablemente a las aplicaciones de pesticidas para el control de plagas, a pesar de la presencia de factores medioambientales favorables para las abejas. En la fase de flores abiertas el registro de abejas por árbol fue relativamente alto debido, evidentemente, a la presencia de un importante numero de flores abiertas con néctar disponible y aromas intensos, así como adecuadas condiciones medioambientales, aunque en menor grado respecto a lo ocurrido en la fase de botoneo, hecho que coincide con lo que afirma INDAP (2016), Di Trani (2007) y Insuasty et al. (2015). Castillo (2002) refuerza lo 
registrado al mencionar que, en palto, la variedad Edranol tuvo mayor cantidad de abejas/ $\mathrm{m} 2 /$ minuto que la variedad Hass, debido a que Edranol tuvo mayor cantidad de flores que el cultivar Hass; sólo en Edranol existió una correlación positiva entre las flores abiertas y la cantidad de abejas.

Tabla 1. Número promedio de abejas Apis mellifera por árbol y por día según fechas de evaluación y fase de la floración de la plantación de mandarino Satsuma Owari en Huaral- Lima, 2015

\begin{tabular}{|c|c|}
\hline $\begin{array}{l}\text { Fecha de evaluación/ } \\
\text { Fase de la floración en la plantación }\end{array}$ & $\begin{array}{c}\text { \# promedio de } \\
\text { abejas por } \\
\text { árbol/día }\end{array}$ \\
\hline 21/09/2015 Botoneo & 1 \\
\hline 25/09/2015 Botoneo & 14 \\
\hline 28/09/2015 Botoneo & 27 \\
\hline 30/09/2015 Botoneo & 24 \\
\hline 02/10/2015 Botoneo & 7 \\
\hline 05/10/2015 Botoneo & 7 \\
\hline 07/10/2015 Botoneo & 18 \\
\hline 09/10/2015 Flores abiertas & 51 \\
\hline 12/10/2015 Flores abiertas & 110 \\
\hline 14/10/2015 Flores abiertas & 100 \\
\hline 16/10/2015 Flores abiertas & 74 \\
\hline 19/10/2015 Flores con pétalos caídos y marchitos & 37 \\
\hline 23/10/2015 Flores con pétalos caídos y marchitos & 20 \\
\hline 26/10/2015 Flores con pétalos caídos y marchitos & 32 \\
\hline 30/10/2015 Flores con pétalos caídos y marchitos & 4 \\
\hline 02/11/2015 Flores con pétalos caídos y marchitos & 8 \\
\hline 04/11/2015 Flores con pétalos caídos y marchitos & 6 \\
\hline 06/11/2015 Flores con pétalos caídos y marchitos & 1 \\
\hline
\end{tabular}

Según Cautín (1996), citado por Castillo (2002), las abejas frecuentan los árboles de palto desde las 11:00 a las 14:00 horas, que es el tiempo en que el estado masculino y femenino de las flores tienen mayor posibilidad de traslape. En la fase de flores con pétalos caídos y marchitos, las bajas poblaciones de abejas registradas obedecen al bajo número de flores con oferta de néctar, a pesar de las buenas condiciones medioambientales existentes. Insuasty et al. (2015) refieren que entre los factores que afectan la frecuencia de visitas de las abejas pecoreadoras están los aspectos climáticos y ambientales, la recompensa floral ofrecida por la planta visitada, la constancia floral hacia cierto tipo de recurso y los factores de atracción de las plantas; durante la mañana y el medio día hay una mayor frecuencia de visitas a la flora que en la tarde.
La Figura 1 muestra las densidades poblacionales de abejas por árbol de mandarino, según fechas de evaluación y fases de la floración.

En la fase de botoneo, el máximo número de abejas por árbol es de 27 el cual, en términos relativos y comparado con lo registrado para otras fases, es bajo y obedecería al escaso número de flores abiertas y predominancia de botones florales, con poca oferta de néctar; aunque, ante la presencia de buenas condiciones climáticas en general. En la fase de flores abiertas, las poblaciones de abejas presentan un incremento bastante importante hasta llegar a alcanzar un pico de 110 abejas por árbol, con un mínimo de 51, situación relacionada a la presencia de importante número de flores abiertas que ofrecen el abundante y atractivo néctar a los insectos polinizadores y en particular a las abejas melíferas. Esto tiene coincidencia con lo que reporta Castillo (2002) en palto, quien indica que la variedad Edranol tuvo mayor cantidad de abejas/m2/ minuto que la variedad Hass, debido a que

Edranol mostró mayor cantidad de flores que el cultivar Hass; sólo en Edranol existió una correlación positiva entre las flores abiertas y la cantidad de abejas. Según Cautín (1996), citado por Castillo (2002), las abejas frecuentan los árboles de palto desde las 11:00 a las 14:00 horas. Por último, en la fase de flores con pétalos caídos y marchitos, el número de abejas desciende progresivamente hasta llegar a niveles de cero abejas por árbol debido a la ausencia de flores abiertas, flores en general y néctar, lo cual coincide con la finalización del periodo de floración, que da paso al cuajado y crecimiento de frutos, lo cual coincide con lo manifestado por Insuasty et al. (2015) quienes afirman que entre los factores que afectan la frecuencia de visitas de las abejas pecoreadoras están los aspectos climáticos y ambientales, y la recompensa floral ofrecida por la planta visitada.

A lo largo del periodo de evaluación, las condiciones climáticas en general evidenciaron ser favorables para la actividad pecoreadora de la abeja melífera excepto el factor horas de sol, el cual, en general, se presentó en número relativamente bajo, habiendo sido nulo en varios momentos por la presencia de neblina, lo cual, de hecho, afectó la actividad de visita de flores y recolección de néctar por parte de las abejas, tal como se aprecia en particular hacia la parte final del periodo 
de evaluación. Repetto (2018), afirma que las condiciones climáticas afectan la actividad de las abejas.

Variación en la densidad de poblaciones de Apis mellifera L., según horarios.
En la Tabla 2 y Figura 2 se presentan los resultados de número promedio de abejas por árbol según los horarios de 8:00 a.m.- 10:00 a.m., 11:00 a.m.- 1:00 p.m. y de 3:00 p.m. - 5:00 p.m., en las fases de botoneo, flores abiertas y flores con pétalos caídos y marchitos.

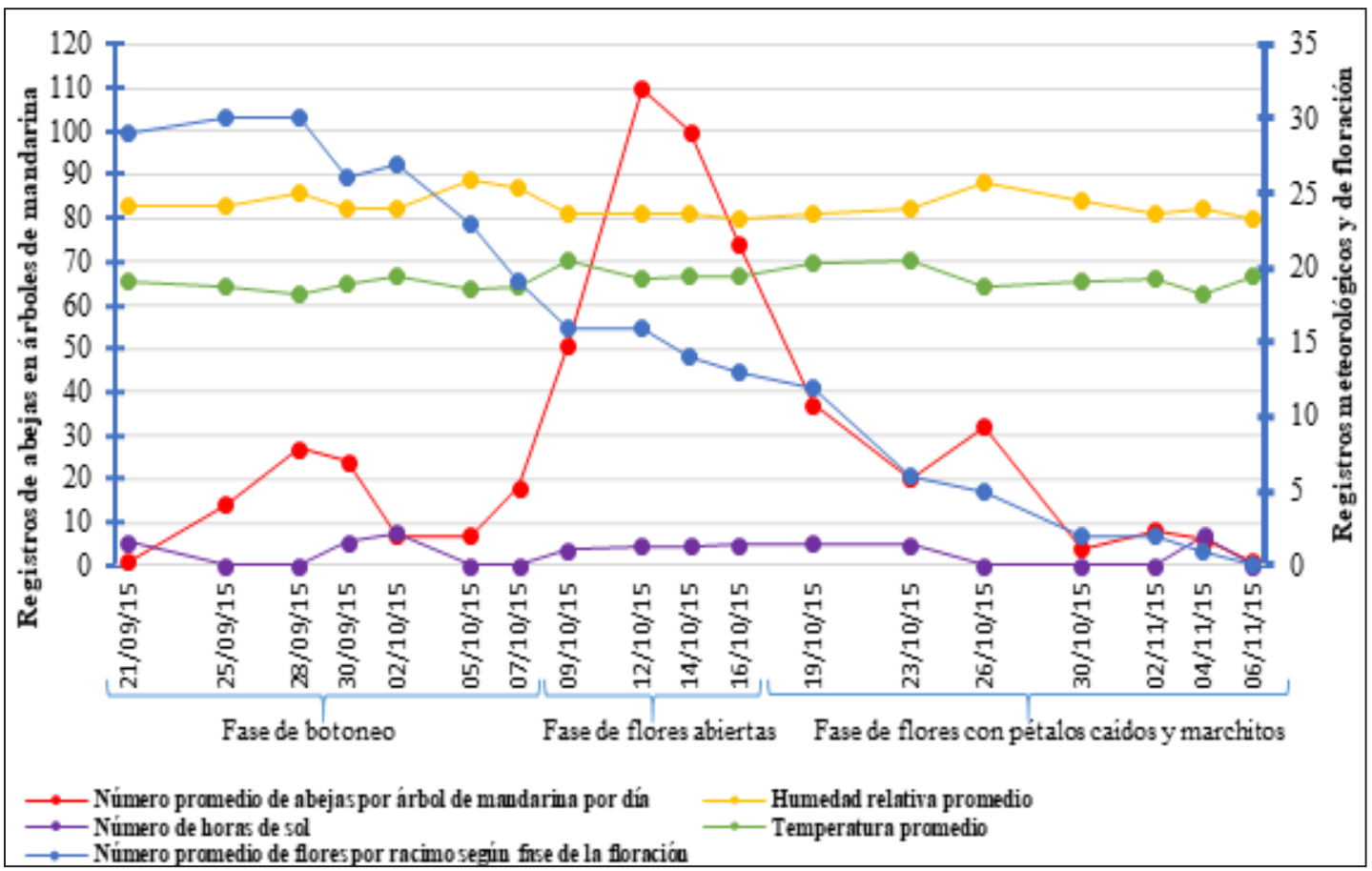

Figura 1. Número promedio de abejas Apis mellifera por árbol y por día según fechas de evaluación y fases de la floración de la plantación de mandarina Satsuma Owari en Huaral - Lima, 2015.

El mayor número de abejas se registró en los horarios de 11:00 a.m.- 1:00 p.m. (232 abejas) y 3:00 p.m. - 5:00 p.m. (227 abejas), en el orden que se indica y con ligeras variaciones numéricas, coincidiendo esto con lo que manifiesta Avellaneda (2009) acerca de la visita de las abejas a las flores de cítricos sucede en el horario de 9 a.m. y 2

p.m. Los menores registros ocurrieron en el horario de 8:00 a.m. - 10:00 a.m. (109 abejas) con una diferencia numérica importante respecto a los anteriores horarios. El mayor número de abejas en los horarios que se indican se debería a las mejores condiciones climáticas presentes en las cuales destaca una adecuada temperatura y luz solar que estimulan el vuelo y las actividades de pecoreo de las abejas, coincidente con lo que refiere Dadant (1979), Osorio et al. (2002) y Insuasty et al. (2015). Por otro lado, los bajos valores en número de abejas en plantas de mandarino registrados en el horario de 8:00 a.m. - 10:00 a.m., estarían sustentados por las condiciones climáticas poco adecuadas y al hecho que las abejas melíferas durante las primeras horas del día y hasta el mediodía despliegan una gran actividad recolectora de polen, no en mandarino pero sí sobre plantas de palto (Persea americana Mill.) e higuerilla (Ricinus communis L.), plantas que producen cantidades apreciables de polen, sobre todo la higuerilla, contrario a lo que ocurre en mandarino cuya producción de polen es casi nula. Esto es corroborado por Castillo (2002) y Di Trani (2007) para palto y melón, respectivamente. Así mismo, durante las mañanas, la intensidad de apertura floral y el aroma de las flores es menor comparado a lo de la tarde. 
Tabla 2. Número promedio de abejas Apis mellifera L. por árbol en tres horarios de evaluación por día, según fechas de evaluación y fases de la floración de mandarina Satsuma en Huaral - Lima, 2015.

\begin{tabular}{|c|c|c|c|}
\hline $\begin{array}{l}\text { Fecha de evaluación/ Fase de la floración de la } \\
\text { plantación }\end{array}$ & $\begin{array}{l}\text { Número promedio de } \\
\text { abejas por árbolde } \\
8 \mathrm{a} 10 \mathrm{~h}\end{array}$ & $\begin{array}{l}\text { Número promedio } \\
\text { de abejas por árbol } \\
\text { de } 11 \text { a } 13 \mathrm{~h}\end{array}$ & $\begin{array}{l}\text { Número promedio } \\
\text { deabejas por árbol } \\
\text { de } 15 \text { a } 17 \mathrm{~h}\end{array}$ \\
\hline 21/09/2015 Botoneo & - & - & 1 \\
\hline 25/09/2015 Botoneo & - & 11 & 3 \\
\hline 28/09/2015 Botoneo & 9 & 11 & 7 \\
\hline 30/09/2015 Botoneo & 6 & 10 & 7 \\
\hline 02/10/2015 Botoneo & 2 & 11 & 7 \\
\hline 05/10/2015 Botoneo & 3 & 11 & 7 \\
\hline 07/10/2015 Botoneo & 5 & 6 & 7 \\
\hline 09/10/2015 Flores abiertas & 8 & 20 & 23 \\
\hline 12/10/2015 Flores abiertas & 14 & 41 & 55 \\
\hline 14/10/2015 Flores abiertas & 22 & 37 & 41 \\
\hline 16/10/2015 Flores abiertas & 17 & 30 & 27 \\
\hline 19/10/2015 Flores abiertas & 10 & 15 & 13 \\
\hline 23/10/2015 Flores con pétalos caídos y marchitos & 2 & 9 & 9 \\
\hline 26/10/2015 Flores con pétalos caídos y marchitos & 6 & 12 & 14 \\
\hline 30/10/2015 Flores con pétalos caídos y marchitos & 1 & 2 & 1 \\
\hline 02/11/2015 Flores con pétalos caídos y marchitos & 3 & 2 & 3 \\
\hline 04/11/2015 Flores con pétalos caídos y marchitos & 1 & 3 & 2 \\
\hline 06/11/2015 Flores con pétalos caídos y marchitos & 0 & 1 & 0 \\
\hline Total: & 109 & 232 & 227 \\
\hline
\end{tabular}

Con relación a las fases de floración, las mayores poblaciones de abejas se registraron en la fase de flores abiertas, seguido por la fase de botoneo y la fase de flores con pétalos caídos y marchitos. Esto pone de manifiesto que el factor que condiciona la presencia de abejas en los árboles de mandarino es la mayor oferta del atractivo néctar e intenso aroma de las flores abiertas, tal como lo señalan Dadant (1979), Castillo (2002) y Santos y Invernizzi (2018). En la fase de botoneo, el número de abejas registradas es numéricamente mayor en el horario de 11:00 a.m. - 1:00 p.m., con valores de 6 a 11 abejas por árbol; en tanto que el menor número de abejas se registró en el horario de 8:00 a.m. - 10:00 a.m. con valores de 2 a 9 abejas por árbol, seguido del horario de 3:00 p.m. - 5:00 p.m. con 1 a 7 abejas. El mayor número de abejas registrado en el horario de 11:00 a.m. - 1:00 p.m., estaría relacionado a condiciones de temperatura y humedad relativa, adecuado brillo solar y a la presencia de olores agradables emitidos por las flores con pétalos abiertos, tal como lo indica INDAP (2016), Osorio et al. (2002) y Gómez y Esteban (2016). Si bien había predominancia de botones florales en este segmento de las evaluaciones, las abejas melíferas acudieron a posarse en ellos, atraídas, probablemente por las sustancias aromáticas que los botones emanan. En los horarios de 8:00 a.m. - 10:00 a.m. y de 3:00 p.m. - 5:00 p.m., además, hubo predominancia de nubosidad y menores valores de temperatura.

En la fase de flores abiertas se registró, en general, valores numéricos importantes de abejas melíferas en los tres horarios. Sin embargo, en los horarios de 3:00 p.m. $-5: 00$ p.m. y de 11:00 a.m. - 1:00 p.m., en ese orden de mérito, hubo una mayor ocurrencia y actividad de abejas, teniendo de 13 - 55 y 15 - 41 abejas por árbol, respectivamente. Por el contrario, en el horario de 8:00 a.m. - 10:00 a.m. se registraron de 8 a 22 abejas por árbol. Estos registros al parecer, obedecen al hecho de que a partir de las 11:00 a.m. hasta las 5:00 p.m. hubo adecuada temperatura y brillo solar, importante ocurrencia de apertura de flores, abundancia de néctar y presencia importante de aromas agradables que las flores emiten por las tardes, lo cual coincide con lo manifestado por VanEngelsdorp y Meixner (2010) y Bacandritsos et al. (2010) quienes determinaron que las abejas visitan las flores de cítricos a lo largo del día y en especial de 2 p.m. a 5 p.m. En la fase de flores con 
pétalos caídos y marchitos, se registró abejas en números relativamente bajos en los tres horarios, en particular en el de 8:00 a.m. - 10:00 a.m. en el cual se registraron de 0 a 6 abejas por árbol, seguido por el horario de 11:00 a.m. a 1:00 p.m. con valores de 1 a 12 abejas por árbol. Sin embargo, los mayores valores se obtuvieron en el horario de 3:00 p.m. - 5:00 p.m., registrándose de 0 a 14 abejas por árbol; aunque el número de abejas registradas en los tres horarios no varían de manera importante entre sí debido a la escasez de flores, en particular flores abiertas, por tratarse de la etapa final de la temporada de floración. Insuasty et al. (2015) indican que entre los factores que afectan la frecuencia de visitas de las abejas pecoreadoras están los aspectos climáticos y ambientales, y la recompensa floral ofrecida por la planta visitada.

La Figura 2 muestra la fluctuación poblacional de $A$. mellifera $L$. en árboles de mandarino en diferentes horarios de evaluación a lo largo del día, según fechas y fases de la floración.

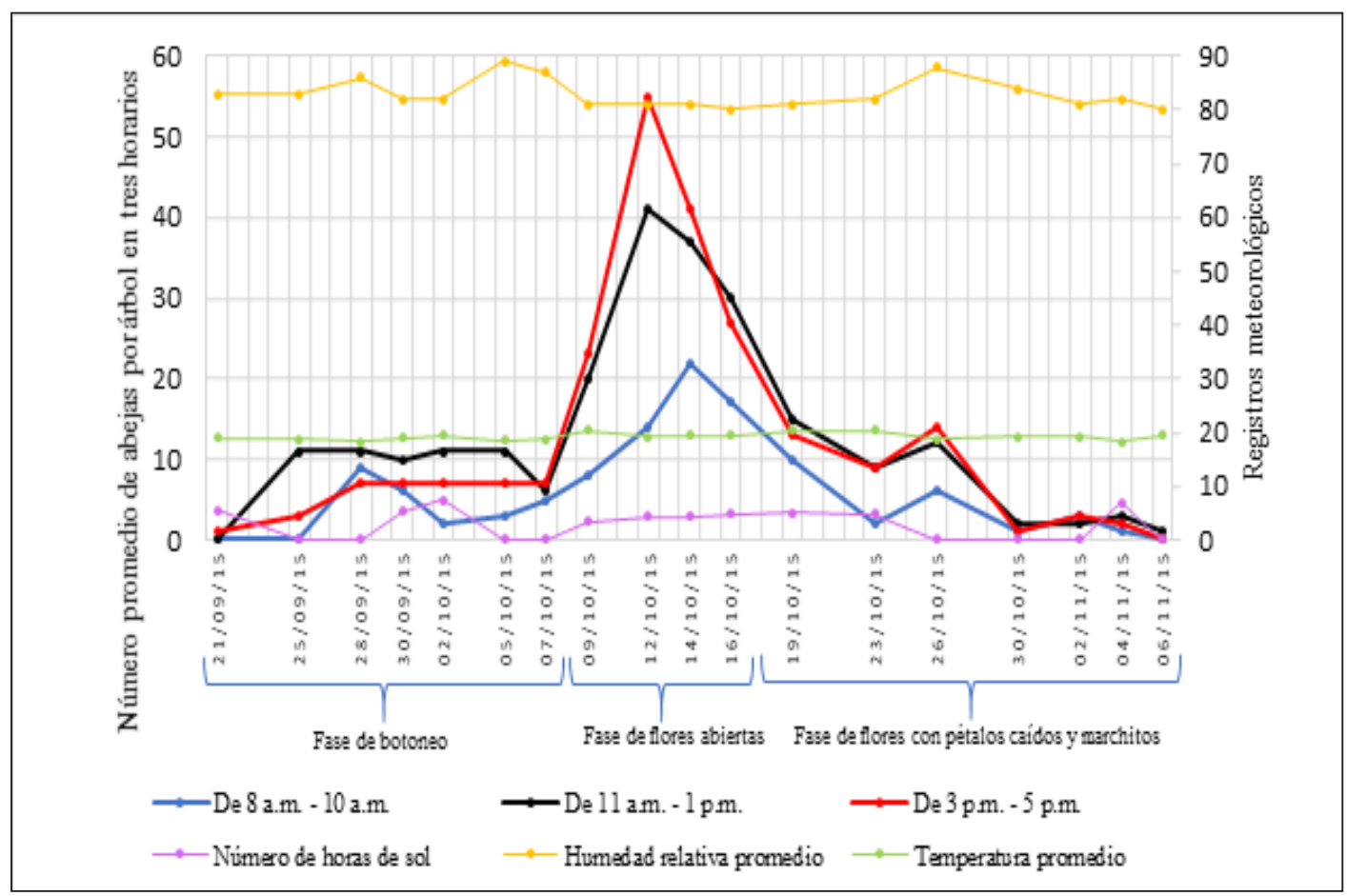

Figura 2. Número promedio de abejas Apis mellifera L. por árbol en tres horarios de evaluación por día según fechas de evaluación y fases de la floración de la plantación de mandarino Satsuma en Huaral - Lima, 2015.

El mayor número de abejas se registró en los horarios de 11:00 a.m.-1:00 p.m. y de 3:00 p.m. - 5:00 p.m., en el orden que se indica y con ligeras variaciones numéricas. Los menores registros ocurrieron en el horario de 8:00 a.m. - 10:00 a.m. con una diferencia numérica importante respecto a los anteriores horarios. Por su parte, las mayores poblaciones de abejas se registraron en la fase de flores abiertas, seguido por la fase de botoneo y la fase de flores con pétalos caídos y marchitos, en los tres horarios de evaluación.
La variación de las poblaciones de abejas melíferas en el horario de 8:00 a.m. - 10:00 a.m. indican que en la fase de botoneo las densidades se mostraron relativamente bajas, las cuales experimentan un incremento moderado hacia la fase de flores abiertas para, posteriormente, en la fase de flores con pétalos caídos y marchitos mostrar valores relativamente bajos de poblaciones de abejas por árbol. En el horario de 11:00 a.m. - 1:00 p.m., en la fase de botoneo, se aprecia que las densidades poblacionales son relativamente bajas, pero numéricamente mayores que aquellas registradas en los horarios de 8:00 a.m. - 10:00 a.m.; 
sin embargo, estas experimentan un incremento importante en la fase de flores abiertas, superando de manera significativa las poblaciones registradas en el horario de 8:00 a.m. - 10:00 a.m., lo cual coincide con reportes dados por Castillo (2002) y Insuasty et al. (2015); mientras que, en la fase de flores con pétalos caídos y marchitos, las poblaciones de abejas decaen a valores relativamente bajos aunque siempre por encima de los valores registrados en el horario 8:00 a.m. - 10:00 a.m., debido probablemente a la presencia de algunas flores abiertas. Las densidades poblaciones registradas en el horario de 3:00 p.m. - 5:00 p.m. muestran valores relativamente bajos en la fase de botoneo, pero por sobre aquellos registrados de 8:00 a.m. - 10:00 a.m., aunque por debajo de los

valores registrados de 11:00 a.m. a 1:00 p.m. En la fase de flores abiertas, experimentaron incrementos importantes en número de abejas por árbol con respecto a lo registrado en los horarios de 8:00 a.m. - 10:00 a.m. y de 11:00 a.m. a 1:00 p.m.; sin embargo, en la fase de flores con pétalos caídos y marchitos, las densidades poblaciones de abejas por árbol muestran valores relativamente bajos, aunque bastante similares a los observados en el horario de 11:00 a.m. - 1:00 p.m., aspecto que coincide con lo manifestado por Insuasty et al. (2015) quienes afirman que entre los factores que afectan la frecuencia de visitas de las abejas pecoreadoras están los aspectos climáticos y la recompensa floral ofrecida por la planta visitada por las abejas. Por otro lado, en flores de melón la actividad colectora diaria de las abejas se inicia poco después de las seis de la mañana, con pocas visitas, y se va incrementando en los períodos siguientes; para el período de 9:30 a 10:30 a.m., se alcanza el clímax de visitas, donde se contabilizan algo más de un cuarto de las visitas diarias, casi todas para la recolección de polen; en el período de 11:00 a.m. a 12:00 m., se experimentó un descenso en la actividad, seguido de un repunte en el siguiente período, de 12:30 a 1:30 p.m.; a partir de ese período, la actividad declina, hasta ser casi nula, llegando a las 5 p.m.; la mayor parte de las visitas, casi la mitad (43\%), fueron realizadas entre 8:00 y 10:30 a.m.; esto puede deberse a que las visitas por polen sólo se realizan hacia las primeras horas de la mañana, entre 7:00 y 11:00 a.m.; las visitas por néctar, en cambio, empezaron desde la mañana, en baja proporción y continuaron hasta entrada la tarde, cerca de las 5:00 p.m. (Di Trani, 2007).
Esta dinámica pone de manifiesto la tendencia de mayor número de abejas por árbol a medida que avanzan las horas a lo largo del día, siendo menores en las primeras horas, moderadas hacia el mediodía y en mayor número por la tarde, habiéndose registrado, además, que las mayores densidades poblacionales ocurren en la fase de flores abiertas por la presencia del néctar en los órganos florales; y menores poblaciones hacia el final del periodo de floración o fase de flores con pétalos caídos y marchitos por la ausencia de flores con disponibilidad de néctar para las abejas; sin embargo, juegan papel importante en la actividad de vuelo y pecoreo de las abejas las condiciones medioambientales como la radiación solar y la temperatura. Lo manifestado coincide plenamente con lo que afirman INDAP (2016), Dadant (1979), Avellaneda (2009), VanEngelsdorp y Meixner (2010) y Bacandritsos et al. (2010).

\section{Comportamiento de Apis mellifera $L$. durante el pecoreo en flores de mandarino.}

La actividad de las abejas empieza con la presencia de los primeros rayos del sol y termina con los últimos haces luminosos; durante este intervalo ocurre la fluctuación de las poblaciones en su actividad de recolección de néctar, polen y agua, lo cual coincide con lo que manifiestan Dadant (1979), Castillo (2002), Di Trani (2007), Avellaneda (2009), INDAP (2016), Repetto (2018) y Santos y Invernizzi (2018).

Las abejas llegan a visitar flores en diferentes fases florales. Así, vistan botones florales blancos y cilíndricos cercanos a la antesis, flores con pétalos parcialmente abiertos, flores con pétalos abiertos y aún flores con pétalos caídos. Antes de tomar contacto con alguna flor, las abejas realizan un vuelo exploratorio en torno al árbol y sobre los racimos florales antes de entrar en contacto directo con la flor. Bajo otras circunstancias las abejas realizan un vuelo que las conduce directamente a la flor. Cuando se trata de una visita a botones florales o flores con pétalos caídos, luego de breve exploración, las abejas se posan sobre la corola aún cerrada en el caso del botón o pueden llegar directamente a la flor con pétalos caídos a la cual inspeccionan muy con brevedad, probablemente para hallar rezagos de néctar. Cuando visitan flores parcialmente abiertas, las abejas usualmente llegan directamente a la zona de apertura, introduciendo parcialmente el cuerpo para acceder, con su proboscis o lengua, al disco nectarífero para succionar el néctar y 
luego alejarse en busca de otras flores. En relación con las flores abiertas, las abejas usualmente llegan de manera directa a la flor dirigiendo sus estructuras bucales al disco nectarífero, a través de la parte basal de los estambres o introduciendo parcialmente el cuerpo desde la parte superior del androceo. En estos dos últimos casos, las abejas, simultáneamente con la recolección del néctar, transfieren polen de su cuerpo al estigma de la flor. Sobre el particular, Palacios (2005), Davies y Albrigo (1999), Machado (comunicación personal, 2015; fundo La Candelaria- Huaral-Lima) y Mendoza (comunicación personal, 2016; fundo La Candelaria-Huaral-Lima) afirman que el mandarino Satsuma es partenocárpico con bajo porcentaje de óvulos fértiles, polen viable pero escaso y frutos que no forman semillas pese a que las flores son visitadas por abejas.

La presencia de abejas sobre botones florales blancos y desarrollados se debería a la atracción que ejercen los aromas de aceites esenciales volátiles y el néctar presentes en el interior del botón, estimándose, por otra parte, que las breves visitas en flores con pétalos caídos y marchitos serían por rezagos de olores de las mismas sustancias antes mencionadas, tal como lo menciona Insuasty et al. (2015). En flores con pétalos abiertos, las abejas se posan en uno de los pétalos y luego se dirigen hacia la parte basal de la misma, abriéndose paso con patas y cabeza por entre los estambres, hasta llegar con su lengua al disco nectarífero para tomar el néctar, acto que la abeja lo repite varias veces en diferentes puntos del disco nectarífero, valiéndose para ello de cortos desplazamientos alrededor de la flor. Este hecho, coincide plenamente con lo expresado por Palacios (2005), en el sentido que las abejas durante el pecoreo se introducen entre los estambres y el gineceo para llegar al disco nectarífero.

Las visitas de las abejas a las flores de mandarino toman un tiempo que varía entre 3 y 20 segundos, tiempo que estaría condicionado a la cantidad de néctar existente en las flores visitadas, lo cual a su vez tendría relación con la hora del día. Las abejas son más numerosas en los racimos florales y permanecen más tiempo en las flores abiertas en horas de la tarde, lo que indicaría que, por cuestiones fisiológicas y climáticas, las flores en ese horario generan una mayor cantidad de néctar, así como también un intenso aroma típico de azahar que fácilmente puede ser percibido. Según Dadant (1979),
Castillo (2002), Di Trani (2007) y Insuasty et al. (2015) el comportamiento de pecoreo de abejas melíferas depende de la cantidad de flores y su contenido en néctar y polen, refiriendo, además, que las abejas melíferas visitan las flores en horarios variables, según especies de plantas, de acuerdo con las horas de secreción de néctar, el cual podría ser más intenso en determinados momentos del día. Por otra parte, en flores de melón, la actividad colectora diaria de las abejas se inicia poco después de las seis de la mañana, con pocas visitas, y se va incrementando en los períodos siguientes; para el período de 9:30 a 10:30 a.m., se alcanza el clímax de visitas, donde se contabilizan algo más de un cuarto de las visitas diarias, casi todas para la recolección de polen; en el período de 11:00 a.m. a 12:00 m., se experimentó un descenso en la actividad, seguido de un repunte en el siguiente período, de 12:30 a 1:30 p.m.; a partir de ese período, la actividad declina, hasta ser casi nula, llegando a las 5 p.m.; la mayor parte de las visitas, casi la mitad (43\%), fueron realizadas entre 8:00 y10:30 a.m.; esto puede deberse a que las visitas por polen sólo se realizan hacia las primeras horas de la mañana, entre 7:00 y 11:00 a.m.; las visitas por néctar, en cambio, empezaron desde la mañana, en baja proporción y continuaron hasta entrada la tarde, cerca de las 5:00 p.m. (Di Trani, 2007). Palacios (2005) manifiesta que las flores de los cítricos son muy atractivas para las abejas, gracias a que despiden un aroma intenso y agradable y producen una alta cantidad de néctar.

En horarios de la tarde el número de abejas en los árboles de mandarino llega a ser tan alto que su zumbido llegar a generar un sonido capaz de ser percibido por el oído humano, sobre todo en zonas, en el campo, con concentración de árboles con alto número de flores como los de cercanías a bordes del campo colindantes con canales de regadío y áreas con mayor grado de humedad del suelo. Se ha podido observar que las flores de mandarino producen cantidades importantes de néctar y escasa o nula cantidad de polen; por tanto, se consideran flores nectaríferas. Las abejas en flores de mandarino Satsuma no muestran actitud de recolección de polen ni formación de pelotillas de polen en las corbículas de las abejas durante la visita en flores de mandarina Satsuma; sin embargo, se observó abejas en flores de mandarino Satsuma con polen en sus corbículas, el cual, al parecer, procede de flores de palto (Persea americana) e higuerilla (Ricinus communis) de 
campos contiguos a la plantación de cítricos, que las abejas visitan en horas de la mañana. Esto coincide con lo encontrado por Castillo (2002) en palto Edranol y Hass. Esto explicaría, en cierto modo, la razón por la que las abejas son más abundantes en plantaciones de mandarino alrededor del mediodía en adelante, luego de su actividad de recolección de polen en otras plantas. En horas de la mañana, fue usual observar una labor muy activa de las abejas sobre flores de Ricinus communis y Persea americana.

Esta apreciación coincide con lo expresado por Machado (comunicación personal, 2015; fundo La Candelaria-Huaral-Lima) quien manifiesta que existe una alta actividad de abejas melíferas en flores de higuerilla entre las 7:00 a.m. - 9:00 a.m. con el propósito de recolectar polen. Lo encontrado en este estudio tiene estrechas coincidencias con lo determinado por Avellaneda (2009) quien observó que la naranja valencia (Citrus cinensis) es visitada por las abejas melíferas entre las 9:00 a.m. y 2:00 p.m. para colectar polen, en tanto que el limón Tahití (Citrus aurantifolia) y al Tangelo (Citrus reticulata x Citrus paradisi) son visitados a lo largo del día, aunque el limón Tahiti es mayormente visitado por las abejas a las 2:00 p.m. y el tangelo principalmente a las 5:00 p.m. En este mismo sentido, en palto, la variedad Edranol tuvo mayor cantidad de abejas $/ \mathrm{m} 2 /$ minuto que la variedad Hass, debido a que Edranol tuvo mayor cantidad de flores que el cultivar Hass; sólo en Edranol existió una correlación positiva entre las flores abiertas y la cantidad de abejas (Castillo, 2002). Así mismo, según Cautín (1996), citado por Castillo (2002), las abejas frecuentan los árboles de palto desde las 11:00 a las 14:00 horas, que es el tiempo en que los estados masculino y femenino de las flores están expuestas.

La investigación permite inferir que la mandarina compite con otros árboles frutales, respecto a la visita de abejas para recolección de polen, néctar y polinización, a partir de las 11 a.m. en temporada de floración.

\section{CONCLUSIONES}

Las poblaciones de Apis mellifera L. en el cultivo de mandarino fueron altas durante la fase de flores abiertas, mientras que en las fases de botoneo y de flores con pétalos caídos y marchitos las densidades fueron relativamente bajas, habiendo sido la presencia de flores abiertas con néctar y agradable aroma, la temperatura y las horas de sol, los factores de mayor influencia. Las mayores poblaciones en árboles de mandarino se registraron en el horario de 11:00 a.m. 1:00 p.m. y de 3:00 p.m. - 5:00 p.m., mientras que en el horario de 8:00 a.m. - 10:00 a.m. las poblaciones fueron menores; sin embargo, los mayores registros siempre ocurrieron en la fase de flores abiertas. El comportamiento de las abejas está marcado por la intensa actividad que desarrollan a lo largo del periodo de floración del mandarino.

\section{Conflictos de intereses}

Los autores firmantes del presente trabajo de investigación declaran no tener ningún potencial conflicto de interés personal o económico con otras personas u organizaciones que puedan influir indebidamente con el presente manuscrito.

\section{Contribuciones de los autores}

Preparación y ejecución: YV, AM, JC; Desarrollo de la metodología: YV, AM, JC; Concepción y diseño: YV, AM, JC; Edición del artículo: YV, AM, JC; Supervisión del estudio: YV, AM, JC.

\section{LITERATURA CITADA}

- Amorós, C. (1999). Producción de agrios. 2 ed. Madrid, ES. Ediciones Mundi- Prensa. 180 pp.

- Avellaneda, K. (2009). Estudio del potencial de Apis mellifera como polinizador para la formación de fruto en un cultivo de naranja (Citrus sinensis) tipo exportación: Caso cítricos del milenio, Bajo Pompeya, departamento del Meta. Trabajo de Grado, Biología. Pontificia Universidad Javeriana. Bogotá - Colombia. 83 pp.

- Bacandritsos, N., Granato, A., Budge, G., Papanastasiou, I., Roinioti, E., Caldon, M., Falcaro, C., Gallina, A., \& Mutinelli, F. (2010). Sudden deaths and colony population decline in Greek honey bee colonies. Journal of Invertebrate Pathology, 105: 335-340.

- Castillo, S. (2002). Efecto de la distancia de las colmenas de abejas (Apis mellifera) a los árboles de palto (Persea americana Mill) y efecto de un segundo ingreso de colmenas de abejas al huerto 
de paltos, sobre el número de abejas encontradas en las flores de palto. Quillota, Chile. 81 pp.

- Dadant, C. (1979). La colmena y la abeja melífera. Montevideo, UY. Editorial Hemisferio Sur. 936 pp.

- Davies, F., \& Albrigo, L. (1999). Cítricos. Zaragoza, ES. Editorial Acribia. 32 pp.

- Di Trani, J. (2007). Visita de abejas (Apis mellifera, Hymenoptera: Apoidea) a flores de melón Cucumis melo (Cucurbitaceae) en Panamá. Rev. Biol. Trop., 55(2):677-680.

- Gómez, I., \& Esteban, M. (2016). El polen apícola como herramienta en el declive de las abejas. Trabajo de Fin de Grado. Facultad de Farmacia. Universidad Complutense. 25 pp.

- INDAP. (2016). Manual Apícola. Editora: Gloria Montenegro. Fac. Agronomía e Ingeniería Forestal. Pontificia Universidad Católica. Chile. $114 \mathrm{pp}$.

- Insuasty, E., Martínez, J., Jurado, H. (2015). Evaluación del proceso productivo apícola, basado en la caracterización etológica de la abeja (Apis mellifera). Veterinaria y Zootecnia, 9(1),115.

- Machado, C. (2015). Comunicación personal. Gerente de campo de mandarinas del fundo La Candelaria - Huaral, Lima.
- Mendoza, M. (2016. Comunicación personal. Empresario agrícola y dueño del fundo citrícola La Candelaria - Huaral, Lima.

- Osorio, T.M., Osorio, T.E., \& Salamanca, G. (2002). Aspectos climáticos relacionados con la actividad apícola tropical. Caso departamento del Tolima.

- Palacios, J. (2005). Citricultura. Tucumán, AR. Editorial Alfa Beta. 518 pp.

- Repetto, J. (2018). Preocupa la pérdida de colmenas en la Argentina. SOBRE LA TIERRA (SLT). Servicio de Prensa y Divulgación Científica y Tecnológica sobre Agronomía y Ambiente. Consultado el 31 de diciembre de 2019.

- Santos, E., \& Invernizzi, C. (2018). Comportamiento de pecoreo de las abejas melíferas en manzanos de las variedades Cripps Pink y Red Chief. Agrociencia Uruguay, 22(2):110.

- VanEngelsdorp, D., \& Meixner, M. (2010). A historical review of managed honey bee populations in Europe and the United States and the factors that may affect them. Journal of Invertebrate Pathology, 103, S80-S95. 